\title{
PROPUESTA DIDÁCTICA PARA EL ESTUDIO CONTRASTIVO DEL LÉXICO EN EL SECTOR DE LENGUAJE Y COMUNICACIÓN*
}

\author{
Liliana C. Belmar Bizama**
}

\section{Resumen}

La enseñanza del léxico es importante para el conocimiento de una lengua, y su uso adecuado muestra la competencia que el hablante tiene de su sistema y de su norma. Por esto, el cambio léxico refleja la idiosincrasia de cada comunidad lingüística. Aquí se propone una unidad didáctica que estudie el cambio semántico, en el campo léxico del vestuario, de manera contrastiva entre el español de España y el español de Chile, y que se aplique a un Tercero Medio en el sector Lenguaje y Comunicación de Enseñanza Media.

Palabras clave: Léxico, cambio semántico, competencia comunicativa, competencia indagativa, unidad didáctica.

\section{Methodological APPROACH TO CONTRASTIVE STUDY OF THE LEXICON IN THE FIELD OF LANGUAGE AND COMMUNICATION}

\begin{abstract}
The lexicon teaching is of paramount importance for a better knowledge of a language, and its suitable use shows the competence that the speaker has of his own system and his norm. The lexical change shows the idiosyncrasy of every linguistic community. Here it is proposed a didactic unit which studies the semantic changes, in the lexical field of the wardrobe, in a contrastive way between the Spanish of Spain and the Spanish of Chile, applied to 11th grade in a school.
\end{abstract}

Keywords: Lexicon, semantic changes, communicative competences, investigative competences, didactic units.

Recibido: 08-05-2014

Aceptado: 23-09-2014

* Texto originado a partir de la Tesis doctoral Propuesta didáctica para el análisis del léxico del español de Chile desde el punto de vista etimológico, L. Belmar, 2011. Universidad Complutense de Madrid, España.

** Chilena, Profesora de Castellano Universidad de Chile, Doctora en Didáctica de la Lengua y la Literatura, UCM, Universidad Metropolitana de Ciencias de la Educación, Santiago, Chile. liliana. belmar@umce.cl 


\section{Introducción}

La adquisición y despliegue de las competencias lingüísticas constituye uno de los puntos clave para el análisis sobre el manejo de la lengua; primordial es, entonces, la educación en el sector Lenguaje y Comunicación, particularmente en el Plan de Formación Diferenciada, ya que busca la mejora del conjunto de conocimientos, habilidades, actitudes y capacidades que permitan a los jóvenes desenvolverse en la sociedad de manera adecuada y competente en las diversas situaciones de la vida cotidiana en la que se valore el uso de la lengua. La asignatura Lenguaje y Comunicación, en la modalidad Científico Humanista, contempla para el nivel de Tercero Medio y del Cuarto Medio de Enseñanza Media un Plan de Formación Diferenciada en el que el alumno puede escoger entre el programa de Literatura e Identidad o el de Lenguaje y Sociedad. Es justamente en este último en donde se destaca el uso de la lengua castellana, especialmente del español de Chile, ya que el Programa de Estudio de este Plan se ha estructurado en tres unidades: Unidad y diversidad de la lengua de la comunidad hispanohablante; El múltiple y cambiante lenguaje de los chilenos; y Algunas jergas de los chilenos.

Como señala la página web del MINEDUC, este programa está centrado en la observación de diferentes situaciones de uso de la lengua castellana con el objeto de que los estudiantes estén más conscientes de su lengua, comprendan mejor cómo funciona, "aprecien la riqueza y variedad de recursos que ofrece y la valoren tanto en cuanto medio de expresión personal, interacción social y creación cultural y artística como en cuanto símbolo de identidad que afirma el sentido de pertenencia a una comunidad" (MINEDUC, 2009). En esta tarea, el docente y la escuela necesitarán seleccionar los contenidos lingüísticos que ayuden a desarrollar la competencia comunicativa de los alumnos y a ordenar y graduar esos contenidos, considerando las necesidades y características del contexto sociocultural de los jóvenes. Este nivel de decisiones requiere no solo de programaciones didácticas generales, sino que también deben tener en cuenta en qué momento, de qué manera, con qué contenidos, con qué materiales didácticos se va a organizar el escenario comunicativo del aula, y también qué y cómo evaluar ese proceso.

La Neurobiología sostiene que el verdadero aprendizaje solo se logra cuando el estudiante le asigna un valor a lo estudiado; de esta manera, el aprendizaje amplía en gran medida las posibilidades que tiene el ser 
humano de adaptarse a muy diversos entornos. Esto es muy claro cuando se trata de la adquisición o conocimiento de una lengua, puesto que "una de las competencias mentales más importantes de la especie humana es el lenguaje hablado que puede iniciar su aprendizaje casi desde la vida intrauterina" (Lavados, 2009: 71). Así es como, al aprender a conocer bien su propia lengua, con sus modismos y características, y a la vez valorar este conocimiento por parte de los alumnos, es que se hacen parte de estos procesos de integración y pertenencia que todo hablante debe tener con su lengua y cultura; ello es parte de su adaptación al medio que lo rodea, puesto que el ser humano

debe adaptarse a los idiomas que se hablan, a las costumbres y creencias, a las culturas, a la geografía, a los recursos naturales, a los esquemas religiosos y valorativos, a las instituciones sociales, al desarrollo tecnológico y al ambiente por éste creado y a cualquier otra característica del entorno que defina competencias necesarias para desempeñarse en la sociedad (Lavados, 2009: 73).

\section{Diseño metodológico}

El profesor de Lenguaje del último ciclo de Enseñanza Media, deberá hacerse cargo del programa "Unidad y diversidad de la lengua de la comunidad", entre cuyos contenidos figura La variedad lingüística de Hispanoamérica y España, y que presenta como aprendizaje esperado, entre otros, que los alumnos "[i]dentifican algunos elementos léxicos, sintácticos, de pronunciación y prosodia que son comunes a la lengua de la comunidad hispanohablante y aquellos en que se manifiestan diferencias regionales" (MINEDUC: 13). Como señala A. de Tezanos (2009) el fin último de la enseñanza es:

la formación de un sujeto culto, poseedor de una caja de herramientas intelectuales y de saberes sociales que le permitan distinguir el ser del deber ser y que, en consecuencia, sea capaz de adquirir compromisos y responsabilidades con el desarrollo de la sociedad en que vive. (169-170)

El programa de Tercero Medio, en una de sus unidades, “El múltiple y cambiante lenguaje de los chilenos", se aboca a la caracterización del español de Chile, tanto en su unanimidad como en su variedad, desde la perspectiva sincrónica y diacrónica. También interesa que valoren el 
dominio del registro culto formal como un aporte a la conservación y unidad de la lengua en el ámbito de la comunidad hispanohablante. Es importante hacer hincapié en la importancia del correcto manejo de la lengua oral y escrita, ya que posibilita que el individuo se supere en todos los ámbitos y, junto con él, el país, porque "El saber y los conocimientos técnicos, las destrezas, habilidades y competencias, en una palabra, los recursos humanos son cada vez más indispensables para la supervivencia de cada país en el mercado mundial" (Arellano, 2000: 14).

Hay tres ejes que se mantienen en toda la trayectoria escolar para el sector de Lenguaje y Comunicación: Comunicación Oral, Lectura y Escritura. Estos se derivan del enfoque curricular del sector al expresar las competencias comunicativas fundamentales que se busca que los alumnos desarrollen (escuchar, hablar, leer y escribir), el desarrollo de la competencia comunicativa es el gran objetivo de esta asignatura. Ello necesariamente implica enriquecer el lenguaje de los estudiantes, ampliando y mejorando la comunicación oral y el acceso al lenguaje escrito a través de la lectura y la escritura. Todo esto se traduce en un enfoque comunicativo funcional que considera el lenguaje como una herramienta eficaz de expresión, comunicación e interacción.

Otro gran objetivo es afianzar la identidad chilena en el uso del lenguaje, y la diferenciación con otras comunidades hispanoparlantes. Este objetivo se desarrolla ya en el último ciclo de EM, en donde se presentan los electivos que se relacionan con el español de Chile, el español de América, etc. y, por supuesto, para su estudio se le da particular énfasis al tema del surgimiento del español (o castellano) como lengua, que se estudia en las unidades de Tercero Medio.

En resumen, este Marco Curricular pone el acento en la adquisición y desarrollo de la competencia comunicativa que permita que los estudiantes, al término de su etapa escolar, se enfrenten con la debida preparación a los desafíos que suponen la continuación de estudios de nivel superior y/o el ingreso al mundo laboral.

Junto con lo anterior, el currículum nacional chileno destaca la necesidad de incentivar el desarrollo de competencias investigativas desde los primeros años de la escolaridad, a fin de consolidar una "cultura de la investigación". De igual forma, la autoridad ministerial se ha encargado de originar instancias adecuadas para que estudiantes y académicos de la Educación Superior opten por este camino. Prueba 
de este interés son los fondos concursables que cada año son asignados a proyectos de ciencia, tecnología, arte y educación. De esta forma, las siglas FONDECYT ${ }^{1}$, CONICYT ${ }^{2}$ y FONDART $^{3}$ se han transformado en referentes familiares, tanto para instituciones como para personas naturales, que ven en estas alternativas de financiamiento la posibilidad de concretar proyectos con intencionalidad formativa.

\section{Objetivos}

A la luz de esta tendencia nacional, se propone una unidad didáctica destinada a implementarse en Tercero Medio que tenga como tema central el estudio comparativo del léxico de la lengua española en dos comunidades: la española y la chilena, puesto que su objetivo final es reforzar la valoración del propio sistema dialectal, a través del conocimiento de las similitudes y diferencias existentes en el uso del léxico, entre el español de España y el español de Chile. Esta unidad pretende ser una contribución al incremento de la competencia comunicativa, la indagativa y la lingüística, capacidades que han de caracterizar al estudiante del siglo XXI. El objetivo esencial de la enseñanza de la lengua y de la literatura es, en la actualidad, la adquisición y el desarrollo de la competencia comunicativa de los alumnos. La educación lingüística y literaria debe beneficiar el desarrollo de las habilidades y competencias de los alumnos, y particularmente debe favorecer la adquisición de la competencia comunicativa y contribuir al dominio de las destrezas lingüísticas básicas y fundamentales. Es la competencia comunicativa la que tiene primordial importancia, puesto que "Las competencias comunicativas son las que posibilitan a una persona actuar utilizando específicamente medios lingüísticos" (MEC, 2002: 9) -tanto es así que en Chile la asignatura que antes se llamaba Castellano ahora se llama Lenguaje y Comunicación-. Esta competencia involucra los códigos lingüísticos y no lingüísticos (semióticos: cinésico, proxémico, icónico, etc.) "la competencia comunicativa comprende varios componentes: el lingüístico, el sociolingüístico y el pragmático" (MEC, 2002: 13), además de la situación particular en que se emplean; se puede decir, entonces, que implica no solo conocer la lengua y su código, sino que el uso de esta en un contexto social determinado. Se puede agregar, que la competencia

\footnotetext{
FONDECYT: Fondo Nacional de Desarrollo Científico y Tecnológico (creado en 1981).

CONICYT: Comisión Nacional de Ciencia y Tecnológía.

FONDART: Fondo Nacional de las Artes.
} 
comunicativa exige a los hablantes de una determinada comunidad lingüística una capacidad cultural que les permita comprender y producir textos, enunciados adecuados a las diversas intenciones comunicativas de su sociedad. Otra competencia que ha adquirido relevancia es la competencia indagativa, que consiste en la capacidad para investigar. Es bien sabido que investigar es la mejor manera de aprender; mediante una investigación, se puede pasar del mundo de la apariencia o de la creencia a aquel donde se dan las verdaderas relaciones entre los diversos fenómenos educativos. La investigación educativa juega un papel muy importante en la actual formulación de políticas educativas, en la administración y en la evaluación de la educación.

\section{Valoración de la norma propia}

Junto con apuntar al desarrollo final de estas competencias mencionadas, así también esta propuesta espera responder a las necesidades culturales que, en la actualidad, se desprenden de la instauración de relaciones globalizadas que se han situado en un lugar importante, restando espacio a las experiencias locales. Frente a esta realidad, parece indispensable generar alternativas que permitan a la sociedad chilena y, especialmente a los jóvenes, recuperar el sentido de pertenencia con respecto a su sistema dialectal. Estas oportunidades deben originarse en la vida académica, puesto que es aquí donde se encuentra la reflexión profunda acerca de las problemáticas que afectan a las distintas comunidades. Tal responsabilidad se relaciona también con la capacidad de reconocer errores de percepción y de uso que pueden incidir, de manera negativa, en la formación de los hablantes cultos, quienes se ven cada día más expuestos a la influencia, no siempre positiva en cuanto al uso del lenguaje -especialmente en el manejo del léxico- de los medios de comunicación de masas y a Internet, pero también a aquellos preconceptos arraigados en esta comunidad lingüística. Un rasgo sociolingüístico particular que se reconoce como propio de los hablantes del español de Chile es su capacidad para dejarse influenciar por los hablantes de otras comunidades de habla hispana que considera prestigiosas, así lo reconoce Salamanca (2010) cuando aclara que esta observación, que es comprobable empíricamente, sin embargo, no aplica de manera homogénea, sino selectiva, pues se produce respecto de aquellas variantes dialectales que se estiman prestigiosas, sea este prestigio explícito o encubierto. Un caso de prestigio explícito es el que 
se evidencia respecto del español de España, en que el hablante de Chile lo revela en actitudes verbales y no verbales (lo declara a nivel de discurso, acepta rápidamente el léxico, pronunciación y entonación de esa variante, cuando debe permanecer algún tiempo en esa comunidad). Lo mismo sucede con la variante argentina, aun cuando sea de prestigio encubierto, es muy fuerte la influencia del habla argentina en el léxico y pronunciación en los hablantes chilenos que viajan a esa nación. Por el contrario, un caso evidente de desprestigio encubierto es el que se presenta con la variante dialectal del Perú, "En efecto, no obstante que muchos chilenos reconocen en los peruanos a "gente que habla bien" -entre otras cosas, 'porque pronuncian todas las eses'- no se sentirían halagados si se dijera de ellos que hablan como peruanos" (Salamanca, 2010), especialmente en estos tiempos en que los peruanos en Chile son mayoritarios en cuanto a inmigrantes de habla hispana.

Estos rasgos de permeabilidad característicos del hablante chileno deben ser considerados por el profesor de Lenguaje y Comunicación, puesto que sus alumnos son hablantes que se están formando, y están sujetos a estas influencias; que valoren positivamente su propia habla, que consideren prestigiosa su propia norma será, en gran medida, tarea del profesor de Lenguaje y Comunicación, quien no solo debe hacerles conocer su sistema lingüístico, sino que, además, apreciar y querer mantener sus rasgos fundamentales, en todos los planos de la lengua.

Un último propósito corresponde a la apreciación de las formas cultas tradicionales chilenas, las que han de constituirse como una significativa fuente, tanto para el conocimiento idiomático como para la apropiación cultural en el contexto globalizado del siglo XXI. Al promover el empleo de este tipo de expresiones, se espera también afianzar en los alumnos una actitud crítica frente a eventuales errores e imprecisiones presentes en textos orales y escritos.

\section{Propuesta}

El objetivo fundamental que persigue esta propuesta va muy acorde con los que plantea el MINEDUC para la asignatura Lenguaje y Comunicación, en el último nivel de Educación Media

Identificar algunos de los usos fonéticos, léxicos, sintácticos y estilísticos propios del castellano de Chile y de otros países americanos hispanohablantes, comparándolos entre sí y con el 
castellano peninsular, analizando el sentido y el valor de estas variantes, así como el valor de la función homogeneizadora de la norma culta. (MINEDUC, 1998: 222)

Una planificación debe contemplar, en primer lugar, la selección de un contenido de aprendizaje. En esta etapa el profesor procede a hacer un análisis de la materia y determina posibles objetivos de aprendizaje como, asimismo, identifica el perfil inicial de los alumnos, de sus representaciones y de sus formas de pensar. Posteriormente, es necesario -además de determinar los objetivos- considerar los obstáculos que podrían impedir el logro de dichos objetivos. Una vez determinado esto, se elabora un dispositivo didáctico que asegure el logro del aprendizaje mediante la determinación del problema, la selección de la situación, de los materiales y del estilo de enseñanza. Al mismo tiempo, el profesor tendrá que considerar estrategias que sirvan de recurso para remediar el problema y que tomen en cuenta la diversidad de los alumnos y las dificultades imprevistas. Estos últimos aspectos solo son verificables a través de una observación continua durante todo el proceso. Finalmente, es necesario tener en cuenta la evaluación del logro de los objetivos como también del progreso intelectual de los alumnos a corto y mediano plazo.

Para cumplir con las metas ya indicadas es indispensable emplear estrategias de aprendizaje que sean congruentes con el modelo curricular constructivista que soporta el ámbito didáctico del currículum actual chileno, y acudir a procedimientos que permitan afianzar la competencia comunicativa y desarrollar, aún más, las habilidades vinculadas con el área de la investigación científica.

Se entiende que una unidad didáctica es un conjunto coherente secuenciado de actividades o tareas planificadas que llevan al alumno, del descubrimiento de elementos y nociones nuevas, a su asimilación, a su fijación y a su transposición. Se concreta en una secuencia de aprendizaje diseñada con la finalidad de que los estudiantes alcancen ciertos objetivos educativos mediante el dominio de contenidos precisos y la realización de determinadas actividades. Para que sea tal, una unidad didáctica debería incluir una presentación o justificación, unos objetivos didácticos, unos contenidos, una secuencia de actividades, una metodología, unas actividades y unos instrumentos de evaluación (Lomas, 2001).

Una vez establecidos los criterios previos a la realización e inicio del curso en que se aplicará esta unidad de trabajo o unidad didáctica, 
es necesario determinar criterios para organizarla. Algunos de ellos, propuestos por Lomas, son: a) afectividad mediante la motivación. Este criterio está destinado a despertar la toma de conciencia del alumno de su rol como autogestor del aprendizaje, del manejo de su tiempo, con el fin de captar la permanente atención de cada estudiante al inicio de cada actividad y de cada clase; b) negociación para poder seleccionar los objetivos y contenidos adecuados, y dar lugar a una organización y reorganización de estos contenidos a lo largo del desarrollo de la unidad. Estos se manifiestan en instancias de evaluación formativa constante; c) selección de objetivos y contenidos, a partir del programa de curso, según las características psicológicas de los alumnos y el carácter de los contenidos (grado de complejidad, de abstracción, etc.); d) secuenciación de tareas y actividades de acuerdo con criterios tales como heurístico, lingüístico, pragmático y sociocultural, referencial y estratégico. Deben abarcar los contenidos conceptuales (qué debe saber el alumno), procedimentales (qué debe saber hacer el alumno) y actitudinales (qué actitudes se deben generar en el alumno); mostrar distintos grados de dificultad; ser flexibles; incluir también algunas tareas voluntarias. Por otro lado, se debe tener en cuenta aquellos contenidos que evoquen otros ya adquiridos por el alumno; e) coherencia: entre objetivos didácticos y terminales, como también entre procedimientos y actividades, y entre contenidos propuestos y adquiridos; f) funcional: debido a que se está desarrollando un tema relativo al uso del léxico en la lengua de una determinada comunidad lingüística, este criterio se aplicará, tomando en cuenta la adscripción al sistema normativo de la lengua; g) adecuación: de las estrategias a la naturaleza a los contenidos, a los materiales y a las condiciones espacio-temporales, socio-antropológicas, cognitivas, etc. h) recursos: estos deben sujetarse tanto a las características de los contenidos que han sido seleccionados, a la metodología que se va a emplear, como a los que están disponibles para el profesor y los alumnos. Pueden ser de muy diversa índole, impresos, orales, visuales, audiovisuales, multimedia, formales, informales, etc. i) evaluación: toda unidad didáctica debe ser evaluada a través de todo el proceso. En este sentido, se deben aplicar distintas modalidades de evaluación (autoevaluación, coevaluación y heteroevaluación). La base metodológica de este criterio se funda en la diversidad y transversalidad de todos los componentes del proceso. Para toda evaluación deben tenerse en cuenta diversas etapas de enseñanza-aprendizaje: motivación, sensibilización, conceptualización y producción. 
La consideración de criterios al momento de planificar resulta indispensable si se quiere garantizar un proceso de enseñanzaaprendizaje en sintonía con las necesidades externas o internas al aula. De esta manera, se ha diseñado un documento que espera entregar los fundamentos procedimentales, tanto de la investigación como de su transposición didáctica al escenario del aula, y se ha concebido como un conjunto de sugerencias facilitadoras de la labor investigativa y no como una guía de ejercicios que ha de ser resuelta con absoluto rigor; por este motivo se omitió su incidencia en la calificación final.

Además, hay que definir el contexto académico en que se aplicará esta unidad, con el fin de lograr de manera óptima los objetivos propuestos, y será necesario, entonces, detallar lo siguiente:

a) Características del contexto educativo. En este sentido, el uso de las TIC es prioridad, puesto que los colegios se han hecho parte del programa nacional del MINEDUC desde 1992; su programa Enlaces ${ }^{4}$ ha contribuido en estos años a reducir la brecha digital en los profesores, que mayoritariamente son "inmigrantes digitales"; esta ha sido una prioridad para Enlaces desde sus inicios ${ }^{5}$. Aun así

existen desafíos pendientes respecto de la formación inicial y continua de los docentes, y a la adquisición de competencias para el uso de las TIC en contextos de aprendizaje [...] como las competencias digitales, y aquellas relacionadas con la búsqueda y selección de información, la comunicación y el trabajo en equipo, el análisis crítico y la resolución de problemas. (MINEDUC, 2011)

Para lograr todo esto, se ha dotado de estas nuevas tecnologías (computadores, software educativos, recursos educativos digitales,

4 Centro de Educación y Tecnología del Ministerio de Educación de Chile. La misión de este Centro es contribuir al mejoramiento de la calidad de la educación por medio de la informática educativa y el desarrollo de una cultura digital en la ciudadanía, con calidad, equidad y pertinencia.

5 Para el logro de este objetivo, en Chile, "la Iniciativa Intel® Educación ha impulsado una estrecha colaboración con instituciones de gobierno, principalmente con el Ministerio de Educación, a través de Enlaces, su Centro Nacional de Educación y Tecnología. El objetivo primordial es apoyar la capacitación de los docentes en el uso pedagógico de las Tecnologías de Información y Comunicación (TIC) fomentando la innovación en los procesos de enseñanza y aprendizaje de los alumnos de educación primaria y secundaria. Asimismo, Intel mantiene vínculos con la Comisión Nacional de Investigación Ciencia y Tecnología (CONICYT) a través del programa Explora, que desarrolla y patrocina a lo largo del año múltiples acciones destinadas a acercar la ciencia y la tecnología a los ciudadanos y con diferentes universidades chilenas para incorporar sus programas en la formación inicial de docentes" (Intel, 2011). 
laboratorios computacionales, etc.) al sistema público de educación, y el 93\% de sus estudiantes ya tiene acceso a las TIC. Unido a ello, en el Marco Curricular se incorporó dentro del currículum Informática en Educación Media, donde el "propósito general del trabajo educativo en Informática es proveer a todos los alumnos y las alumnas de las herramientas que les permitirán manejar el 'mundo digital' y desarrollarse en él en forma competente" (MINEDUC, 1998).

b) Características del grupo-curso, cómo está constituido, nivel académico de los alumnos, etc.; todo ello con el objeto de tratar de nivelar y homogeneizar al grupo desde el comienzo.

c) Características particulares de su motivación por el sector y por la asignatura misma, los problemas y desafíos que tienen frente a este tipo de contenidos, y otros. También se debe tener en cuenta el enfoque que tendrá este estudio dentro del sector, los contenidos, la metodología, los criterios para la evaluación, etc.

\section{Actividades sugeridas}

A continuación se ofrecen algunas actividades que pueden facilitar un estudio semántico desde la perspectiva contrastiva. Si bien es cierto que no todas ellas son obligatorias, y por tanto no todas tienen incidencia en la calificación final del curso, se recomienda su desarrollo secuencial, para así lograr los objetivos propuestos.

\subsection{Investigación sociolingüística}

La determinación de los elementos históricos y sociales que afectaron o afectan a los cambios semánticos en las comunidades lingüísticas de España y de Chile, se llevará a efecto a través del análisis de textos escritos literarios y no literarios que reflejen el campo léxico (Coseriu, 1991) del vestuario. Aquí se delimita el corpus y se seleccionan las formas que se contrastarán. El resultado de este estudio puede presentarse en una línea del tiempo, que es muy didáctica y sinóptica, además que permite clasificar los eventos importantes y establecer una cronología del cambio en cada forma estudiada.

En lo referente al contraste español de España/español de Chile, serán admitidos también aquellos testimonios procedentes de registros audiovisuales que se encuentren asociados al campo léxico escogido. En este caso, se sugiere la revisión de mensajes publicitarios presentes en 
periódicos y revistas, videos, espacios radiales y televisivos, búsqueda en internet e incluso en escenarios urbanos (vitrinas, medios de transporte, etc.), para dar con todo tipo de contextos y situaciones comunicativas.

Es importante señalar, además, que la validez de los registros obtenidos estará determinada, en gran parte, por el empleo de instrumentos lexicográficos. De esta forma, la utilización de diccionarios onomasiológicos, de chilenismos, americanismos, panhispánicos, y el Corpus de referencia del español actual (CREA) que la Real Academia Española de la Lengua ofrece en línea, brindará a la investigación mayor consistencia científica, aportando antecedentes que no solo permiten analizar los aspectos lingüísticos de un determinado fenómeno semántico, sino que también apreciar sus implicancias comunicativas. Esta búsqueda bibliográfica puede ser de textos impresos, pero también con los que están en la red, y que pueden consultarse de manera gratuita.

\subsection{Búsqueda de ejemplos correspondientes al campo léxico del vestuario}

En este punto se deben considerar clasificaciones que permitan organizar las formas revisadas en grupos acotados a partir de un rasgo distintivo claramente especificado: tipo de vestuario, uso formal/ informal, usuario (hombre/ mujer, niño/adulto, etc.). Con esto se pretende facilitar la exposición de los datos, lo cual favorecerá tanto a los investigadores como a los eventuales destinatarios del informe final del estudio. Para realizar esta búsqueda de ejemplos, se puede acudir a los registros audiovisuales que entregan la televisión, internet, la radio, los periódicos y revistas. A modo de ejemplo, se presenta una muestra contrastiva de los usos en las comunidades de hablantes seleccionadas (española y chilena). Estas muestras, unidas a la imagen presentada, facilitan la pesquisa de los rasgos diferenciales existentes en el significado y uso dado a las formas estudiadas. Los resultados de esta búsqueda pueden presentarse usando organizadores gráficos que muestran los resultados de la investigación de manera visual y sinóptica, lo que refuerza su comprensión. Además, ayudan a procesar, ordenar, recordar y priorizar la información lo que facilita la comprensión de lo estudiado. Aquí se puede usar, por ejemplo, el Mindmap que permite un trabajo colaborativo en clases, lo que resulta muy atractivo para los alumnos, quienes también pueden elaborar mapas lingüísticos con los resultados, en donde se reflejen las isoglosas delimitadas. 


\subsection{Estudio de casos. Búsqueda de informantes que proporcionen los datos}

Esta actividad, referida a la oposición español de Chile/español de España, propone la realización de entrevistas a informantes de ambas comunidades. Las entrevistas pueden registrarse con Windows MovieMaker y para ello se requiere de la cámara web del computador para capturar video. También funciona con dispositivos de grabación de audio independientes. Esta herramienta permite separar las pistas de audio y video para agregar música o una voz en off a la grabación. Con esta actividad, se puede destacar el uso del léxico en su contexto real, la frecuencia, nivel en que se utiliza, etc. Para esta instancia los investigadores-alumnos deben manejar una muestra representativa y equilibrada de informantes.

\subsection{Análisis comparativo del uso del léxico en español de Chile y el español de España}

Esta instancia requiere el empleo de diversos corpus en ambas variantes, documentos que registren los usos orales y escritos de formas pertenecientes a estas dos comunidades lingüísticas, como es el caso del CREA 6 . Se otorga valor a estos registros, porque en ellos es posible visualizar el vocablo en su contexto cotidiano o usual, lo cual no necesariamente se aprecia en otros instrumentos lexicográficos. De esta manera, el alumno puede distinguir las situaciones comunicativas en las que estas voces son aplicadas, lo que brinda también referencias acerca del compromiso afectivo del hablante con su lengua. Como una forma de sintetizar la comparación efectuada, se propone la elaboración de un esquema contrastivo, tal como el "Circep" o concepto circular, o puede ser con una presentación multimedia, cuya aplicación es motivadora puesto que es clara, efectiva, creativa e innovadora si no se exagera su utilización en clases. Las más usuales son Microsoft PowerPoint, que no necesita conexión a Internet, y Prezi, que está en línea.

6 La Real Academia Española de la Lengua desde 1993 trabaja en un banco de datos del español. El registro sincrónico, el Corpus de Referencia del Español Actual (CREA), recoge el uso contemporáneo (desde 1975) del español y sus variantes en todas las comunidades de habla hispana. Este uso se documenta en un conjunto de textos de diversa índole, ya sean literarios, prensa, revistas, etc. tanto escritos como orales, y que son almacenados en soporte informático para ser estudiados, tanto en su significado como en su contexto. Actualmente, cuenta con 140 millones de registros, pero se espera llegar a los 160 millones. 


\subsection{Desarrollo de textos auténticos que incorporen formas del campo léxico estudiado}

En este punto, se pretende que los alumnos observen y apliquen el uso correspondiente a cada comunidad, poniendo especial énfasis al uso particular chileno. Para lograr esto, se sugiere que trabajen con textos literarios, medios de comunicación (orales, escritos y audiovisuales) y textos informativos concretos que les permitan no solo observar el uso, sino también el contexto y la frecuencia en que se da ese uso por parte de cada comunidad lingüística. Una vez establecidos los rasgos diferenciales y los unificadores entre las comunidades, los alumnos podrán elaborar sus propios textos con el léxico estudiado. Para compartir estos textos y elaborarlos en grupo, hay una herramienta muy útil que es la Google Docs que permite crear documentos en línea con el grupo, construir en colaboración el texto, archivarlo, editarlo, etc. y, además, se puede acceder a la conexión desde el computador, el teléfono móvil o un Iphone.

\subsection{Discusión de los resultados}

Esta etapa del trabajo del curso se requiere de la participación activa y constante de los estudiantes. Puede ser una actividad de discusión presencial, realizada en clases, como también puede ser de manera virtual, en línea. La ventaja de este formato es que no necesariamente es sincrónica, puede ser asincrónica, lo que también ayuda a la reflexión. Para esta modalidad se requiere del uso de una plataforma virtual con fines educativos; hay varias, pero una que es gratuita, efectiva y cuyo entorno de aprendizaje está basado en los principios pedagógicos constructivistas es la plataforma Moodle, porque, entre otras cosas, permite compartir los aprendizajes, construirlos de manera colaborativa, y propicia la participación activa del alumno. Asimismo es una herramienta útil para el profesor, porque le permite asignar tareas en línea, entregar de manera diversa la información, llevar un registro del curso, tener un calendario de acontecimientos próximos, exponer las calificaciones, enviar correos, archivar todos los documentos, la comunicación se agiliza y puede ser personalizada con cada estudiante, con el grupo y entre los mismos alumnos. Entre las formas de establecer este contacto está el blog (cuaderno de trabajo o bitácora que puede registrar comentarios a los que se da respuesta y se establece un diálogo, posteriormente), la charla (permite el intercambio asincrónico privado entre el profesor y un alumno o entre dos alumnos), el chat (comunicación 
en tiempo real), el foro (permite realizar un intercambio asincrónico del curso sobre un tema compartido, la participación del alumno en un foro puede ser una parte integral de su experiencia de aprendizaje, ya que lo ayuda a aclarar y desarrollar su comprensión del tema), la consulta (se crea una pregunta con opciones de respuesta, esto permite ir evaluando de manera instantánea o realizar votaciones rápidas sobre un tema). Todo esto ayuda de manera significativa al logro de los objetivos de la investigación, puesto que se comparten los resultados, hay discusión de los temas, integración y participación activa en todo momento.

\subsection{Planificación de un trabajo que se presente en forma oral}

Esta última instancia se ha concebido como el espacio que permite a los alumnos integrar todos los aspectos del trabajo realizado, enriqueciéndolos con elementos que proceden del área de la didáctica. De este modo, se ha contemplado la presentación de una exposición, cuya elaboración se oriente hacia el cumplimiento de objetivos claramente definidos (la evolución semántica de palabras relativas al vestuario y que debe contemplar el empleo de instrumentos lexicográficos) y que representen el desarrollo de aprendizajes significativos acerca de las variedades idiomáticas y sus rasgos esenciales.

\section{Presentación de la unidad}

Para planificar y presentar esta unidad se sugiere el empleo de modelos de planificación adscritos al paradigma constructivista, como es el Modelo $T$, dado a conocer por Martiniano Román, especialista en temas curriculares ${ }^{7}$, quien considera la figura del alumno como eje de todo el proceso educativo. Por esta razón se brinda protagonismo a tres componentes de la experiencia de aprendizaje: los conceptos, las capacidades y las actitudes que se incorporarán o consolidarán como conocimiento, una vez concluido el proceso educativo.

7 “Trata de integrar los elementos básicos del currículum (capacidades y valores como objetivos y contenidos y métodos - procedimientos como medios), en una sola hoja para que sea percibido de una manera global y desde ella el profesor pueda construir una imagen mental útil para su actuación profesional. Una vez identificados los elementos básicos del currículum, pretende facilitar su desarrollo" (Román, 2001: 72).

"Se denomina Modelo T, porque tiene forma de doble T: la T de medios (contenidos y métodos / procedimientos) y la T de objetivos (capacidades - destrezas y valores - actitudes)" (Román, 2001: 73). 
Una segunda instancia corresponde a los medios, entendidos como herramientas que apoyan el desarrollo de un determinado aprendizaje. Tales recursos son tangibles (rotuladores, textos, computadores, pizarras, etc.) e intangibles (formas de hacer, pasos para lograr el desarrollo de capacidades, aprender contenidos, valores, actitudes).

La última parte de este modelo se ha reservado a la evaluación, llevada a efecto tanto por el profesor como por el alumno. En el primer caso, se deben consignar evaluaciones diagnósticas, procesuales y acumulativas; en el segundo, la evaluación de los pares (coevaluación) y la apreciación del alumno frente a su propio desempeño (autoevaluación), los registros de estas evaluaciones se recogerán de las rúbricas que el profesor haya diseñado para cada actividad. El objetivo de este trabajo de investigación que realizarán los alumnos, bajo la orientación y supervisión del profesor, es que se produzca en ellos un cambio motivacional, un aprendizaje conceptual y procedimental de las técnicas de investigación en el campo de la lingüística hispánica y un incremento en su rendimiento en relación con el aprendizaje del tema estudiado. Se presenta a continuación un esquema que sintetiza lo descrito anteriormente, y que permitirá visualizar la unidad preparada. 


\section{Objetivos}

Objetivo Fundamental vertical

- Distinguir el carácter evolutivo de la lengua, registrado en el campo léxico del vestuario, en las variantes de español de España y español de Chile.

- Relacionar la diversidad cultural como causa del cambio semántico y con hitos del desarrollo cultural de los pueblos.

Objetivos por capacidades y valores

- Reconocer la influencia de la comunidad lingüística en la fijación del léxico.

- Analizar de manera creativa el uso propio de la comunidad a la que se pertenece.

- Evaluar críticamente el cambio semántico en la lengua.

Objetivos por destrezas y actitudes

- Representar de manera esquemática y original los rasgos unificadores y los diferenciadores de las variantes estudiadas

- Precisar los significados particulares de las variantes estudiadas, respetando los usos de cada comunidad.

- Aplicar adecuadamente los instrumentos lexicográficos, por medio de la observación atenta de sus particularidades.

Objetivo Fundamental Transversal

- Valorar críticamente el cambio semántico como manifestación cultural y como parte de la idiosincrasia nacional, fortaleciendo el respeto por las variantes dialectales. 


\begin{tabular}{ll}
\hline Contenidos & Procedimientos \\
\hline 1. Semántica y Lexicología & 1. Lectura comprensiva de textos lingüísticos. \\
- objeto de estudio & 2. Trabajo bibliográfico. \\
- enfoques & 3. Búsqueda de informantes y / o ejemplos en \\
- sincronía y diacronía & textos o medios de comunicación. \\
- unidades léxicas & 4. Análisis comparativo. \\
- recursos léxicos & 5. Elaboración de una síntesis gráfica. \\
- campo léxico & 6. Investigación etimológica. \\
2. La lexicografía & 7. Búsqueda lexicográfica de ejemplos \\
- definición y descripción & relativos al campo léxico en diversos tipos \\
- instrumentos lexicográficos & de diccionarios (chilenismos, americanismos, \\
3. Cambio semántico & panhispánicos, etc.). \\
- causas & 8. Desarrollo de textos auténticos que incorporen \\
- clasificación & formas correspondientes al campo léxico \\
- denotación y connotación & estudiado. \\
- relaciones semánticas &
\end{tabular}

\section{Actividades}

1. Investigación sociolingüística acerca de los cambios semánticos.

2. Elaboración de una línea del tiempo.

3. Búsqueda y localización de ejemplos de usos correspondientes al campo léxico seleccionado y su relación con los aspectos culturales estudiados. 4. Entrevista a hablantes de las dos normas dialectales del español y recolección de ejemplos de usos correspondientes al campo del vestuario. 5. Producción de un moviemaker que refleje las entrevistas lingüísticas.

6. Realización de organizadores gráficos que muestren los resultados.

7. Elaboración de mapas lingüísticos que muestren las isoglosas.

8. Análisis comparativo, determinando rasgos diferenciadores, que se plasmen en un Circep o exposición de una presentación multimedia que muestre de manera comparativa los resultados.

9. Selección de formas pertenecientes al campo léxico, para determinar su evolución etimológica, utilizando como fuente diccionarios especializados.

10. Discusión de los temas trabajados, en forma presencial y a distancia.

11. Planificación de una presentación oral. 


\begin{tabular}{|c|c|c|}
\hline \multicolumn{3}{|c|}{ Evaluación } \\
\hline Diagnóstica & Procesual-formativa & Sumativa \\
\hline $\begin{array}{l}\text { - Uso del vocabulario } \\
\text { adecuado y expresión } \\
\text { oral correcta en una } \\
\text { presentación individual } \\
\text { breve. } \\
\text { - Lectura comprensiva de } \\
\text { un texto lingüístico. } \\
\text { - Interrogación oral breve } \\
\text { al grupo curso al principio } \\
\text { del semestre y al iniciar } \\
\text { cada clase. } \\
\text { - Elaboración de protocolos } \\
\text { clase a clase. }\end{array}$ & $\begin{array}{l}\text { - Verificar que cada } \\
\text { alumno y /o cada } \\
\text { grupo reciba (y dé) } \\
\text { retroalimentación sobre la } \\
\text { eficacia en la ejecución de } \\
\text { las tareas y el trabajo en } \\
\text { equipo. } \\
\text { - Confirmar que los } \\
\text { alumnos y los grupos } \\
\text { reflexionen sobre la } \\
\text { retroalimentación que } \\
\text { reciben. } \\
\text { - Guiar a los alumnos en } \\
\text { la selección de las TIC } \\
\text { para cada actividad. } \\
\text { - Ayudar a los individuos } \\
\text { y a los grupos a delimitar } \\
\text { objetivos para mejorar su } \\
\text { trabajo. } \\
\text { - Observaciones del } \\
\text { profesor durante el } \\
\text { trabajo en equipo. } \\
\text { - Alentar a los alumnos } \\
\text { a que festejen el buen } \\
\text { desempeño. }\end{array}$ & $\begin{array}{l}\text { - Administración del } \\
\text { tiempo. } \\
\text { - Presentaciones de los } \\
\text { trabajos en clase. } \\
\text { - Exámenes escritos. } \\
\text { - Aplicación de los } \\
\text { conceptos a una situación. } \\
\text { - Evaluación de los demás } \\
\text { miembros del equipo, } \\
\text { de la contribución de } \\
\text { cada uno de ellos para el } \\
\text { proyecto. }\end{array}$ \\
\hline
\end{tabular}

- Habilidad para emitir opiniones - Organización del tiempo. $\begin{array}{lll}\text { personales y puntos de vista. } & \text { - Constancia y perseverancia en los } \\ \text { - Capacidad para dialogar y estudios. }\end{array}$

argumentar. - Participación en clases.

- Habilidad para defender su punto - Cantidad de ayuda ofrecida a los de vista. miembros del equipo.

- Habilidad para no dominar la - Esfuerzo personal acorde con las discusión. exigencias del curso.

- Evaluación de la contribución en el proyecto de grupo.

- Cantidad de ayuda ofrecida a los miembros del equipo. 


\section{Conclusiones}

Como consecuencia de los cambios curriculares que derivan de la Reforma Educacional que se ha implementado en Chile desde 1996, el estudio del español de Chile, que nunca antes había formado parte de los contenidos de la asignatura de Lenguaje y Comunicación -antes Castellano-, ha pasado a ser un contenido central en el nivel de Tercer Año de Enseñanza Media Científico-Humanista Diferenciado.

Elobjetivo de este trabajo de investigación que realizarán los alumnos, bajo la orientación y supervisión del profesor, es que se produzca en ellos un cambio motivacional para conocer más de su lengua, un aprendizaje conceptual y procedimental de las técnicas de investigación en el campo de la lingüística y un incremento en su rendimiento en relación con el aprendizaje del tema estudiado.

Para lograr este objetivo se propuso la aplicación del diseño curricular Modelo T, que permite la visualización completa de la unidad programada y aborda todos los aspectos que son relevantes en una planificación, puesto que exige considerar los contenidos en sus tres dimensiones: conceptuales, procedimentales y actitudinales, y atiende a diversos objetivos (verticales, transversales, específicos); junto a todo ello, requiere pensar cómo se logrará el aprendizaje, es decir, la metodología que se empleará. Esta Propuesta se ajusta al modelo constructivista y, en este sentido, al planificarla, usando este modelo $\mathrm{T}$, por sus características, hace que resalten dichos postulados constructivistas (que enmarcan el currículum educacional chileno), ya que su estructura apunta preferentemente a la forma de adquirir las habilidades por parte del alumno, a través de las actividades propuestas centradas en los estudiantes; es decir, permite diferenciar claramente los contenidos de las competencias que deben desarrollarse y estimularse en los alumnos, y que ellos, a su vez, se hagan cargo de manera activa de su propio aprendizaje. Los alumnos son el eje central en esta propuesta, lo que se refleja en las múltiples estrategias y actividades que se han planteado para que sean desarrolladas de manera individual y colectiva, asumiendo ellos así un papel central en el proceso; de esta manera, los estudiantes podrán edificar su aprendizaje y desarrollar las habilidades, actitudes y valores que se postulan para este estudio del léxico.

En cuanto a las actividades propuestas, se destaca el hecho de que se incorporan en ellas las que provienen del ámbito de las $\mathrm{TIC}$, puesto 
que su conocimiento y uso corresponden a competencias que se quiere desarrollar. El uso de las TIC y de Internet en la enseñanza-aprendizaje puede ser provechoso, porque aporta metodologías innovadoras, puede mejorar la planificación didáctica de la clase y apoya a la docencia presencial y no presencial, lo que redunda en favorecer el trabajo autónomo del estudiante fuera del aula, por mencionar algunas ventajas.

La propuesta también promueve y estimula el trabajo colaborativo del grupo al impulsar la realización de varias actividades en conjunto y también que los resultados de las investigaciones personales se presenten ante el curso. Con ello se pretenden fomentar ciertas habilidades sociales tales como pedir y aceptar la ayuda del otro, el respeto mutuo, la tolerancia y comprensión, la disposición para escuchar, poder coevaluar y aceptar la evaluación de los pares.

\section{Referencias bibliográficas}

Arellano, J. P. (2000). Reforma Educacional: prioridad que se consolida. Santiago de Chile: Editorial Los Andes.

Coseriu, E. (1991). Principios de semántica estructural. Madrid: Gredos.

Intel. (2011). Iniciativa Intel® Educación Chile. Recuperado de <http: / / www.intel.com/education/la/es/paises/chile/>

Lavados, J. (2009). La Neurobiología del Aprendizaje y la Educación. Estudios Sociales, 117, 69-90.

Lipman, M. (1997). Pensamiento complejo y educación. Madrid: Ed. de la Torre.

Lomas, C. (2001). Cómo enseñar a hacer cosas con las palabras. Teoría y práctica de la educación lingüística. Vols. I y II. Barcelona: Ed. Paidós.

MEC. Instituto Cervantes. (2002). Marco común europeo de referencia para el aprendizaje, la enseñanza y la evaluación de las lenguas. Recuperado de <http:/ / cvc.cervantes. es.obref/marco>

MINEDUC. (2009). Objetivos Fundamentales y Contenidos Minimos Obligatorios de la Educación Media. Recuperado 
de <http://www.curriculum- mineduc.cl/.../ Marco_Curricular_Ed_Basica_y_Media_ Actualizacion_2009.pdf>

MINEDUC. (2009). Propuesta de ajuste curricular. Recuperado de <http:/ / www.curriculum-mineduc.cl / ayuda / docs / ajustecurricular-2/Sector_Lenguaje_y_Comunicacion. pdf>

MINEDUC. (2011). Enlaces - Centro de Educación y tecnología. Recuperado de $<$ http://www.enlaces.cl/index. php?t=44\&i=2\&cc $=1296 \& \mathrm{tm}=2>$

MINEDUC. (1998). Objetivos Fundamentales y Contenidos Mínimos Obligatorios de la Educación Media. Santiago de Chile.

Ministerio de Educación, República de Chile. (2001). Lenguaje y Sociedad. Programa de Estudio de Tercer o Cuarto Medio, Formación Diferenciada. Santiago de Chile.

Real Academia Española: Banco de datos (CREA). (s.f). Corpus de referencia del español actual. Recuperado de $<\mathrm{http}$ / / www.rae.es>

Román, M. y E. Díez López. (2001). Diseños curriculares de aula. Un modelo de planificación como aprendizaje-enseñanza. Buenos Aires: Ediciones Novedades Educativas.

Salamanca, G. (2010). Apuntes sociolingüísticos sobre la presencia de argentinismos en el léxico del español de Chile. Atenea, 502, 125-149.

Tezanos, A. De. (2009). La Identidad de la Profesión Docente: Una mirada desde la acreditación. Estudios Sociales, 117, 149-180. 